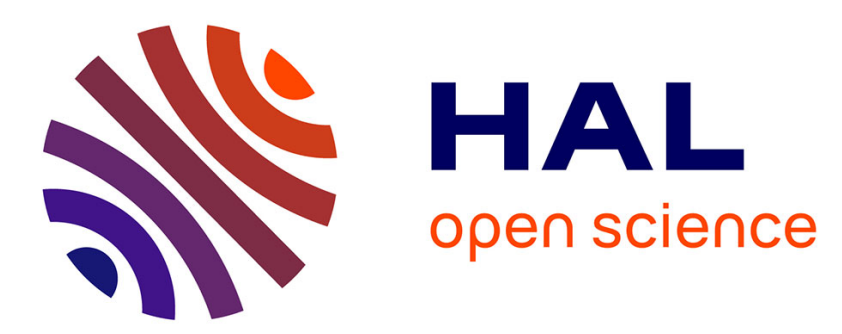

\title{
Elasticity and yielding of calcite paste: scaling laws in a dense colloidal suspension
}

Teresa Liberto, Marie Le Merrer, Catherine Barentin, Maurizio Bellotto, Jean Colombani

\section{- To cite this version:}

Teresa Liberto, Marie Le Merrer, Catherine Barentin, Maurizio Bellotto, Jean Colombani. Elasticity and yielding of calcite paste: scaling laws in a dense colloidal suspension. Soft Matter, 2017, 13 (10), pp.2014-2023. 10.1039/C6SM02607A . hal-02051876

\section{HAL Id: hal-02051876 https://hal.science/hal-02051876}

Submitted on 28 Feb 2019

HAL is a multi-disciplinary open access archive for the deposit and dissemination of scientific research documents, whether they are published or not. The documents may come from teaching and research institutions in France or abroad, or from public or private research centers.
L'archive ouverte pluridisciplinaire HAL, est destinée au dépôt et à la diffusion de documents scientifiques de niveau recherche, publiés ou non, émanant des établissements d'enseignement et de recherche français ou étrangers, des laboratoires publics ou privés. 


\section{Journal Name}

\section{ARTICLE TYPE}

Cite this: DOI: $10.1039 / \mathbf{x x x x x x x x x x}$

\section{Elasticity and yielding of calcite paste: scaling laws in a dense colloidal suspension}

\author{
Teresa Liberto*a ${ }^{* a}$ Marie Le Merrer ${ }^{a}$, Catherine Barentin ${ }^{a, c}$, Maurizio Bellotto ${ }^{b}$ and Jean \\ Colombani $^{a}$
}

Received Date

Accepted Date

DOI: 10.1039/xxxxxxxxxx

www.rsc.org/journalname

\begin{abstract}
We address the mechanical characterization of calcite paste as a model system to investigate the relation between microstructure and macroscopic behavior of colloidal suspensions. The ultimate goal is to achieve a control of the elastic and yielding properties of calcite which will prove valuable in several domains, from paper coating to paint manufacture and eventually in the comprehension and control of the mechanical properties of carbonate rocks. Rheological measurements have been performed on calcite suspensions for a wide range of particle concentrations. The calcite paste exhibits a typical colloidal gel behavior, with an elastic regime and a clear yield strain above which it enters a plastic regime. The yield strain shows a minimum when increasing the solid concentration, connected to a change of the power law scaling of the storage modulus. In the framework of the classical fractal elasticity model for colloidal suspensions of Shih et al. [Phys. Rev. A, 1990, 42, 4772], we interpret this behavior as a switch with the concentration from the strong-link regime to the weak-link regime, which had never been observed so far in one welldefined system without external or chemical forcing.
\end{abstract}

\section{Introduction}

Calcite is the most stable polymorph of calcium carbonate $\left(\mathrm{CaCO}_{3}\right)^{1}$ and an extremely widespread mineral. It is found in many igneous, metamorphic and sedimentary rocks as an accessory mineral and it can also have biochemical origin. Moreover, calcite is the principal constituent of limestone, chalk and marble, extremely common rocks and one of the largest carbon repositories on our planet ${ }^{2}$. The mechanical and optical properties of calcite and its availability make it one of the most widely used minerals as a construction material, abrasive, agricultural soil treatment, extender for pigments, excipient for pharmaceuticals and more. In many uses like filler for paper manufacture or building materials, calcite is ground to powder and dispersed in aqueous solutions, resulting in the formation of a paste, i.e. a yield stress fluid, solid-like under low stresses but flowing at large stresses ${ }^{3-5}$. However, most of the previous works on calcite paste rheology have focused on the influence of organic additives on flow properties, driven by applications in cement and paper industries, while the elasticity and yielding of pure calcite paste remain almost unexplored.

\footnotetext{
${ }^{a}$ Institut Lumière Matière, Université de Lyon, Université Claude Bernard Lyon 1, Domaine scientifique de la Doua, 6 Rue Ada Byron, 69622 Villeurbanne cedex (France)

${ }^{b}$ Dipartimento di Chimica, Materiali ed Ingegneria Chimica "G. Natta", Politecnico di Milano, Piazza Leonardo da Vinci 32, 20133 Milano (Italy)

${ }^{c}$ Institut Universitaire de France (France)

*E-mail:teresa.liberto@univ-lyon1.fr
}

Typical calcite particles are found in the range of microns if obtained from grinding or tens to hundreds of nanometers if obtained by precipitation, and can thus be considered as colloids. Microscopically, in presence of attractive interactions, colloids aggregate into fractal flocs. For a sufficiently high concentration, the flocs interconnect and form a network called gel, similar to the network of blobs in polymeric gels, which confers the paste an elastic-like behavior ${ }^{6}$. The elastic properties of the suspension depend on those of the flocs and on the links between them. Shih et al. ${ }^{6}$ and subsequently $\mathrm{Wu}$ and Morbidelli ${ }^{7}$ have proposed a model to describe the power-law dependence of the mechanical properties of colloidal gels versus the solid concentration based on the floc structure and organization, which has been successfully used to interpret the elastic and plastic behaviors of many such gels ${ }^{8-19}$. One of the main predictions of this model is that, when the colloid concentration increases, a transition in the mechanical properties of the material is expected between two scaling laws. Surprisingly, despite the widespread utilization of this acclaimed model, this central feature has never been observed experimentally in the absence of external or chemical forcing.

We use conventional rheology to characterize both the elastic and yielding behavior of calcite colloidal gels, for volume concentrations varying in the range $5 \% \leq \phi \leq 30 \%$. At $\phi \approx 20 \%$ we evidence a striking change in the dependency upon concentration of the yield strain, separating the elastic and plastic regimes, correlated to a change in the power law dependence of the elastic 
modulus upon concentration. These results constitute a complete validation of the above model of the mechanics of colloidal suspensions in the full range of concentrations ${ }^{6}$, whereas up to now only parts of the model were used for one well-defined unforced system.

This article is organized as follows. In section 2, sample preparation, rheological and optical measurements are described. In section 3, after ruling out the effect of slippage as a rheological artefact, the influence of calcite concentration on elasticity and yielding is studied in detail. In section 4, the results are discussed and analysed along the model of Shih et al. ${ }^{6}$, which allows to characterize the microstructure of the calcite paste, i.e, the fractal dimension and the size of the flocs constituting the paste as a function of the concentration.

\section{Materials and Methods}

\subsection{Sample preparation}

A calcite dense colloidal suspension was obtained by dispersion of Socal 31 calcite powder (Solvay, average particle diameter $60 \mathrm{~nm}$, density $\rho=2710 \mathrm{~kg} / \mathrm{m}^{3}$ ), in distilled water. The range of volume concentrations is $\phi=5-30 \%$. The volume concentrations are deduced from the powder weight and calcite density. Sample preparation was carried out in a vortex stirrer (Ultra Turrax TD300). The homogenization process required five minutes mixing at an increasing mixing rate (4500-5800 rpm) on increasing volume concentration. Immediately after mixing, the suspension appears white because it scatters light due to the mismatch of refractive index between water and calcite. The strong scattering suggests the existence of heterogeneities at the micrometric level, larger than the initial nanometric particles ${ }^{20}$. The $\mathrm{pH}$-range of the calcite suspensions, measured on fresh paste, slightly increases with $\phi$ in the range 8.7-8.9, typical for calcite water suspensions ${ }^{21}$.

\subsection{Rheological Measurements}

The viscoelastic properties were determined using oscillatory deformation applied by a stress-controlled rotational rheometer (Anton Paar MCR 301). The measurements were performed in a plate-plate (PP) geometry at room temperature. Upper and lower plate diameters respectively of $36 \mathrm{~mm}$ and $64 \mathrm{~mm}$ were used. The dimension of the gap, in the range $0.5-5 \mathrm{~mm}$, was chosen much bigger than the average diameter of the nanoparticles and of the heterogeneities (i.e. flocs), in the aim to obtain bulk rheological properties (Fig. 1a). More precisely, we studied the influence of the gap width ranging from 0.5 to $5 \mathrm{~mm}$ on the local deformation field and on the rheological measurements. Four repeated measurements were performed for every concentration, with a new sample loading for each measurement.

To characterize the viscoelastic response of the paste, we extract from the measurements the storage modulus $\left(G^{\prime}\right)$ and the loss modulus $\left(G^{\prime \prime}\right)$, defined as follows ${ }^{22}$ :

$$
G^{*}=G^{\prime}+i G^{\prime \prime}
$$

where $G^{*}$ is the complex modulus corresponding to the ratio be- tween the complex stress $\tau^{*}$ and complex strain $\gamma^{*}$ :

$$
\tau^{*}(t)=G^{*} \gamma^{*}(t)
$$

The entire experimental protocol consists in a preparation phase and a measurement one. The preparation phase includes two steps: pre-shear and time structuration. The pre-shear consists in a 1 minute imposed shear rate of $\dot{\gamma}=10 \mathrm{~s}^{-1}$, in order to start from comparable initial conditions for each sample. The time structuration is a 5 minutes small amplitude oscillation step at imposed deformation $\gamma_{0}=0.01 \%$ and frequency $f=1 \mathrm{~Hz}$. During this resting time, the sample restores the colloidal interactions that were destroyed in the pre-shear step and thus recovers rigidity, as indicated by the increase of the storage modulus $G^{\prime}$ which reaches a constant plateau after one minute. The measurement phase is an amplitude sweep in oscillatory regime at imposed frequency ( $f=1 \mathrm{~Hz}$ or $f=10 \mathrm{~Hz}$ ), with increasing deformations in the range: $\gamma_{0}=0.001-10 \%$ or increasing stress in the range: $\tau=1-300 \mathrm{~Pa}$. The two different amplitude sweeps give equivalent results, although the stress amplitude sweep is less noisy, since we work with a stress-controlled rheometer. During this step, the values of the stress and strain are acquired by the rheometer, from which the viscoelastic moduli are computed.

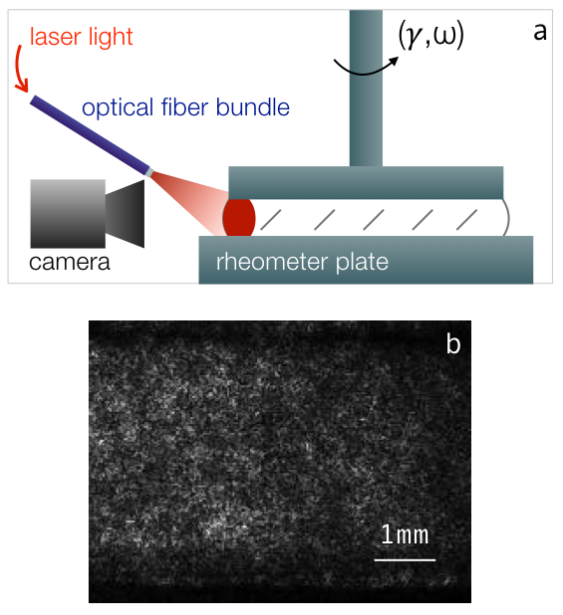

Fig. 1 (a) Sketch of the experimental setup. (b) Image of the sample with the speckle pattern created by the light backscattered from the sample.

\subsection{Data analysis}

At low strains, up to the end of linearity, we find that the elastic modulus $G^{\prime}$ is typically one order of magnitude larger than the loss modulus $G^{\prime \prime}$, revealing the elastic-like behavior of the paste (Fig. 2). Besides, at small deformations, the system reacts linearly such that the storage and loss moduli are constant. In this plateau regime, the linear storage modulus $G^{\prime}$ lin can thus be estimated unambiguously.

At larger strains, the material yields and becomes liquid-like, and both moduli drop dramatically (Fig. 2). The purpose of our paper is to explore in detail the gel destructuration, i.e. the 


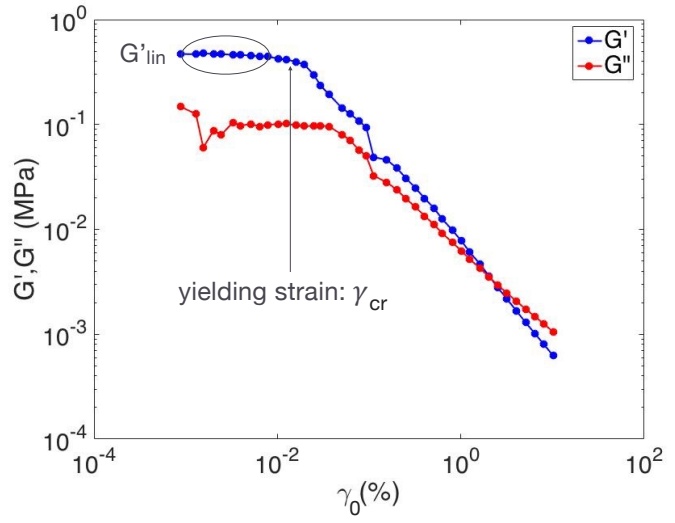

Fig. 2 Typical amplitude sweep measurement $(\phi=20 \%, f=1 \mathrm{~Hz}, 46 \mu \mathrm{m}$ rough sand paper plates): storage $G^{\prime}$ and loss $G^{\prime \prime}$ moduli as a function of the deformation $\gamma_{0}$.

onset of plasticity. To characterize it, we estimate the end of the linear regime by a critical strain $\gamma_{\mathrm{cr}}$, which corresponds to the value of $\gamma_{0}$ for which the storage modulus is $10 \%-15 \%$ lower than the value in the elastic regime $G_{\text {lin }}^{\prime}$. We also denote $\tau_{\mathrm{cr}}$ the corresponding critical stress.

\subsection{Shear characterization with Diffusing-Wave Spec- troscopy}

Since our samples are highly turbid and strongly scatter light, we have also used an optical probe based on Diffusing-Wave Spectroscopy (DWS) techniques ${ }^{23}$ to characterize the local shear dynamics across the rheometer gap. Coherent light from a HeNe laser (wavelength $\lambda_{0}=632 \mathrm{~nm}$ ) is sent onto the side of the rheometer cell thanks to an optical fiber bundle. The illuminated zone is a few millimeters wide and we image the sample surface from the side with a camera (IDS UI) and objective. The experimental setup is shown in Fig. 1a.

A speckle interference pattern is created (Fig. 1b), whose temporal evolution reveals the relative motion of light scatterers, hence the inner dynamics of the sample, namely the shear oscillations. Videos are made during stress sweep experiments similar to the ones used for the elasticity measurements at $\phi=20 \%$. The sample oscillation is $f=10 \mathrm{~Hz}$, while the video acquisition frequency is fixed at $50 \mathrm{~Hz}$, with an exposure duration of $20 \mathrm{~ms}$. To analyse the recordings, we use time and space resolved correlation spectroscopy ${ }^{24-26}$. After background subtraction, we first define rectangular areas of interest (AOI) of area $20 \times 200$ square pixels (approximately $0.6 \times 6 \mathrm{~mm}^{2}$ ) at different positions $z$ across the gap. For each AOI, we calculate at each time the following autocorrelation function:

$$
F(t, \Delta t, z)=\frac{2\left\langle I_{t} I_{t+\Delta t}\right\rangle-\left\langle I_{t}\right\rangle^{2}-\left\langle I_{t+\Delta t}\right\rangle^{2}}{\left\langle I_{t}^{2}\right\rangle+\left\langle I_{t+\Delta t}^{2}\right\rangle-\left\langle I_{t}\right\rangle^{2}-\left\langle I_{t+\Delta t}\right\rangle^{2}}
$$

where $I_{t}$ is the pixel intensity at time $t,\langle\ldots\rangle$ denotes the average over the AOI and $\Delta t$ is a delay time, which is an integer multiple of the time interval between successive frames ( $\Delta t=n \times 20 \mathrm{~ms})$.
In the following, we only consider the case $\Delta t=20 \mathrm{~ms}$.

In the absence of motion inside the sample, $F$ is equal to 1 , but its value decreases as the light scatterers are sheared past each other. For a simple shear oscillatory flow, the DWS theory predicts $^{27-29}$ :

$$
F(t, \Delta t, z)=\exp \left[-2 k \ell^{*} \Gamma|\Delta \gamma| \sqrt{2 / 5}\right]
$$

where $\Gamma \approx 2$ is a numerical prefactor, $k$ is the light wavenumber in the medium (here water), $\ell^{*}$ is the light transport mean free path and $\Delta \gamma=\gamma(t+\Delta t)-\gamma(t) \approx \dot{\gamma}(t) \Delta t$ is the change in deformation during the delay $\Delta t$. If we now write that the strain varies periodically, i.e., $\gamma=\gamma_{0} \cos (2 \pi f t)$, and further assume that $\Delta t f \ll 1$ (slow dynamics compared to the delay $\Delta t$ ), we can simplify the above expression to

$$
\begin{aligned}
F(t, \Delta t, z) & =\exp \left[-4 \pi \sqrt{2 / 5} k \ell^{*} \Gamma \gamma_{0} f \Delta t|\sin (2 \pi f t)|\right] \\
& \approx 1-4 \pi \sqrt{2 / 5} k \ell^{*} \Gamma \gamma_{0} f \Delta t|\sin (2 \pi f t)|
\end{aligned}
$$

in the limit $k \ell^{*} \gamma_{0} \ll 1$. The time and space resolved autocorrelation function $F$ thus provides a measurement of the absolute value of the actual shear deformation rate inside the sample. $F$ thus oscillates with time and its average over one period $T=1 / f$ reads:

$$
F_{0}(z)=\langle F(t)\rangle_{T} \approx 1-8 \sqrt{2 / 5} k \ell^{*} \Gamma \gamma_{0} f \Delta t
$$

$F_{0}(z)$ enables us to check the homogeneity of the deformation along $z$, as detailed below. Besides, the sensitivity of the method to the amplitude of the shear oscillations $\gamma_{0}$ is for instance revealed in Fig. 3, which corresponds to a stress sweep experiment, for which we calculate the spatial average of $F_{0}$ :

$$
F_{m}=\left\langle F_{0}(z)\right\rangle_{z}
$$

Fig. 3a shows $F_{m}$ as a function of time, while Fig. 3b shows the corresponding values of imposed stress and apparent deformation extracted from the rheological measurements. As increasing stresses are applied to the paste, the deformation increases, and the measured autocorrelation value $F_{m}$, starting from the value $\approx 1$, decreases step by step (Fig. 3a), until a more important drop (at $\approx 160 \mathrm{~s}$ ), corresponding to the onset of the liquid-like behavior, emphasized by the sharp increase in deformation $\gamma_{0}$ (Fig. 3b). The optical data thus allow us to estimate the variation of local strain, which can be compared to the macroscopic strain applied by the rheometer geometry.

\section{Results}

In this section, we first characterize the elasticity and the yielding of the calcite paste for a given concentration. In particular, the effect of slippage of the paste is analyzed and ruled out. Other rheological artefacts ${ }^{30}$ such as flow inhomogeneity and sample aging are also discussed. Then the influence of the paste concentration on the elastic modulus and on the yielding point is studied in detail. 

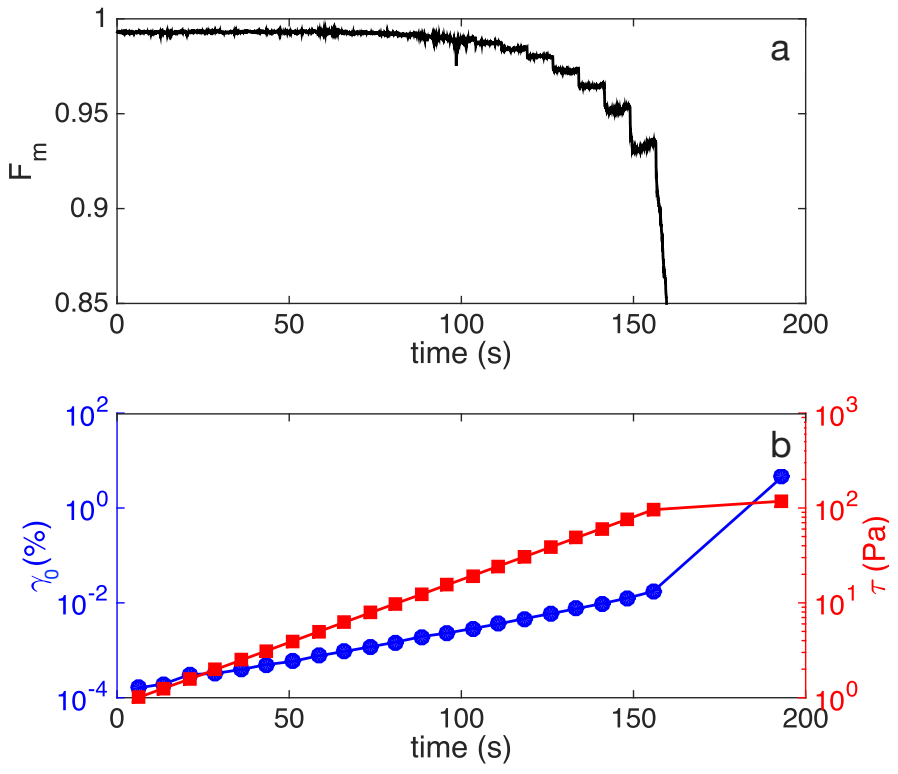

Fig. 3 Typical stress sweep experiment: (a) optical signal $F_{m}$ and (b) rheological data (applied strain, blue circles and stress, red squares) as a function of time. $F_{m}$ denotes the value of $F(t, \Delta t)$, for $\Delta t=20 \mathrm{~ms}$, spatially averaged and smoothed over one oscillation period $(0.1 \mathrm{~s})$ to extract the continuous component of the oscillating signal. The calcite volume concentration is $\phi=20 \%$ and the gap width is $4 \mathrm{~mm}$ with $20 \mu \mathrm{m}$-rough PMMA plates.

\subsection{Influence of surfaces and gap width}

An important aspect to investigate is the wall aptitude to drag the paste. In general, to prevent slippage, a rough surface is recommended. The choice of roughness should be adequate to provide sufficient grip, keeping an accurate and well defined geometry, without secondary flows ${ }^{31}$. In our case, we tested four different surfaces: sand papers with typical roughness of $162 \mu \mathrm{m}$ and $46 \mu \mathrm{m}$ and PMMA smooth and with roughness $20 \mu \mathrm{m}$. If the surface prevents slippage we expect the local rheological quantities (stress and strain) to be independent of the gap. We thus tested the different surfaces at various gap widths, from 0.5 to $5 \mathrm{~mm}$.

Fig. 4 shows the variation of the estimated $G_{\text {lin }}^{\prime}$ and $\gamma_{\mathrm{cr}}$ as a function of the gap width, for the different surfaces and $\phi=20 \%$. $G^{\prime}$ lin first increases with the gap width, then saturates for widths larger than $\approx 3 \mathrm{~mm}$. In the same time, $\gamma_{\text {cr }}$ decreases, then also saturates. This gap-dependency suggests that slip at the plate walls might be non-negligible for gaps smaller than $3 \mathrm{~mm}$. For a given applied stress, we expect a combination of the actual deformation of the sample $\gamma_{\text {real }}$ and slip at the walls. If we denote $\delta_{\text {top }}$ and $\delta_{\text {bottom }}$ the slip distance on the top and bottom plates of the rheometer, the deformation imposed by the rheometer is $\gamma_{\text {app }}=\gamma_{\text {real }}+\left(\delta_{\text {top }}+\delta_{\text {bottom }}\right) / h$ where $h$ is the gap width. Hence, the larger the gap, the smaller the contribution of slip to the total deformation, which is consistent with the measurements of Fig.4.

This effect seems more pronounced for sand papers than for PMMA. This could be due to specific interactions between calcite and silica (sand) or PMMA ${ }^{32,33}$. To evidence the presence of

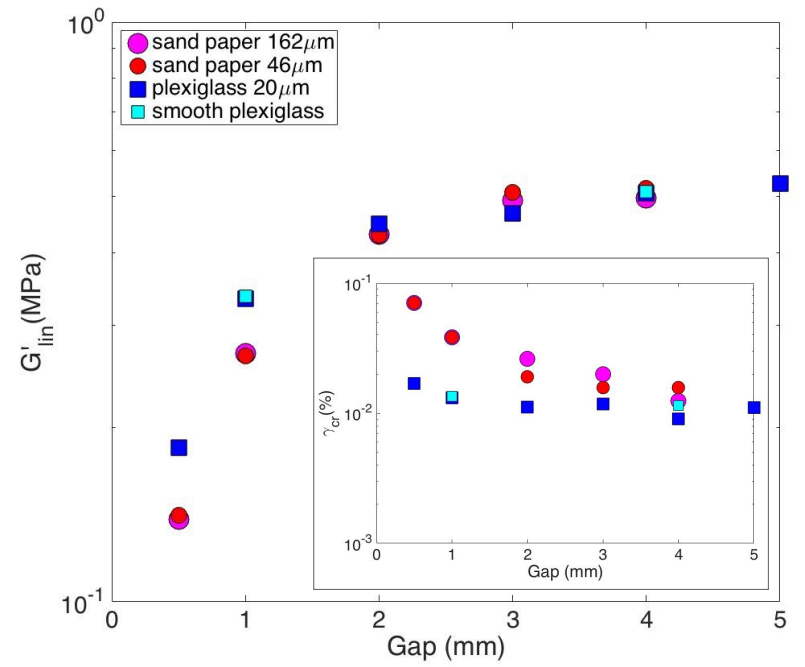

Fig. 4 Linear storage modulus $G^{\prime}$ lin as a function of the gap width for different surfaces for a volume concentration of $\phi=20 \%$ and frequency $f=1 \mathrm{~Hz}$. The standard deviation for the $G^{\prime}$ lin values is $15 \%$. In the inset is represented the critical strain $\gamma_{\mathrm{cr}}$ at the same conditions with a standard deviation less than $25 \%$.

slip at smaller gaps, we performed optical DWS measurements to compare the deformation inside the sample at two different gap widths ( 1 and $4 \mathrm{~mm}$ ). Figure 5 shows the optical signal $F_{m}$ (averaged spatially and over one oscillation period), as a function of the apparent deformation imposed by the rheometer, for the 2 different gap widths. For each gap, two experiments are shown to illustrate the reproducibility of the optical measurement. For the $4 \mathrm{~mm}$ gap, we find that $F$ decreases roughly linearly with the strain applied by the rheometer $\gamma_{0}^{\text {app }}$, as expected from Eq.6. On the contrary, for the $1 \mathrm{~mm}$ gap, the optical decorrelation is much reduced, indicating that the actual deformation in the sample is lower than expected from the motion of the rheometer plate: this is characteristic of slip of the paste at the walls, thus confirming our interpretation of Fig. 4.

Consequently, all the measurements shown below have been performed with sand paper plates (roughness $46 \mu \mathrm{m}$ ) at a gap width of $4 \mathrm{~mm}$, except for pastes of low concentrations $(\phi \leq 10 \%)$. Such pastes cannot remain in large gap cells, and a smaller gap width of $1 \mathrm{~mm}$ have then been used, always checking the independence of the data upon gap (see appendix).

\subsection{Deformation homogeneity}

At large gaps ( $4 \mathrm{~mm}$ ), the optical DWS signal can be calculated at different positions across the gap, in order to check the deformation homogeneity. More precisely, we extract the value of $F(t, \Delta t=20 \mathrm{~ms}, z)$ for different positions $z$ and average it over one oscillation period to obtain the continuous component $F_{0}$. Figure 6 shows the variation of $F_{0}$ as a function of $z$, for 3 different strains $\gamma_{0}$, in the solid-like regime of the paste, for the experiment of Fig. 3. The origin of $z$ is taken at the top plate. We find that $F_{0}$ decreases as $\gamma_{0}$ increases, as seen above. Besides, we notice that $F_{0}$ is independent of the position $z$, thus ruling out shear banding effects in the elastic regime ${ }^{34}$. Note that we have taken 


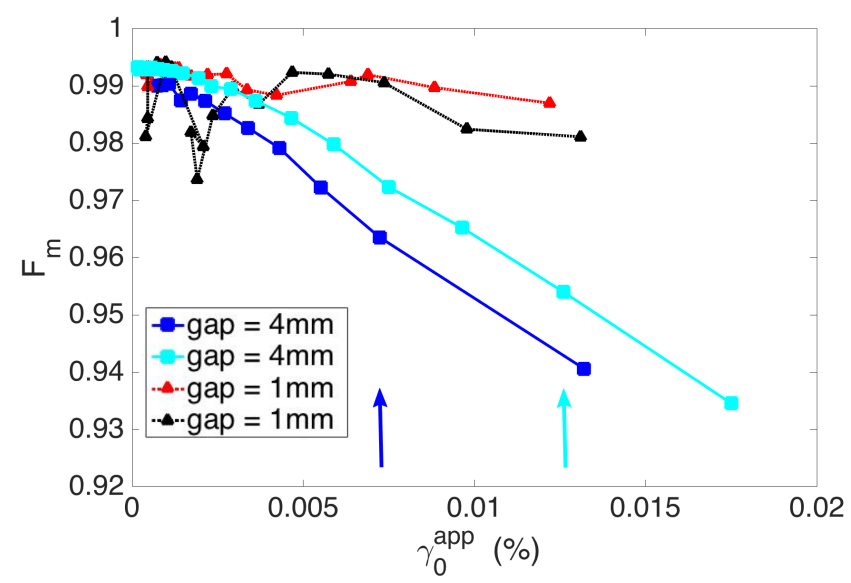

Fig. 5 Comparison between optical and rheological measurements: optical signal $F_{m}$ as a function of the strain applied by the rheometer $\gamma_{0}^{\text {app }}$, for two different gap widths: $1 \mathrm{~mm}$ (red and black triangles) and $4 \mathrm{~mm}$ (blue and light blue squares). The arrows indicate the end of the elastic regime for $4 \mathrm{~mm}$ gap experiments. The calcite volume concentration is $\phi=20 \%$ and the experiments are performed with $20 \mu \mathrm{m}$-rough PMMA plates.

care here to illuminate the sample as homogeneously as possible, to get a clean optical signal in the whole gap. However, we have also noticed that, in the liquid-like regime, conversely to the solidlike one, shear localization occurs, as can be directly seen on the picture of Fig. 6(c), where the deformed region appears blurred, because in this case the temporal evolution of the speckle pattern is much faster than the camera exposure time $20 \mathrm{~ms}^{35}$.

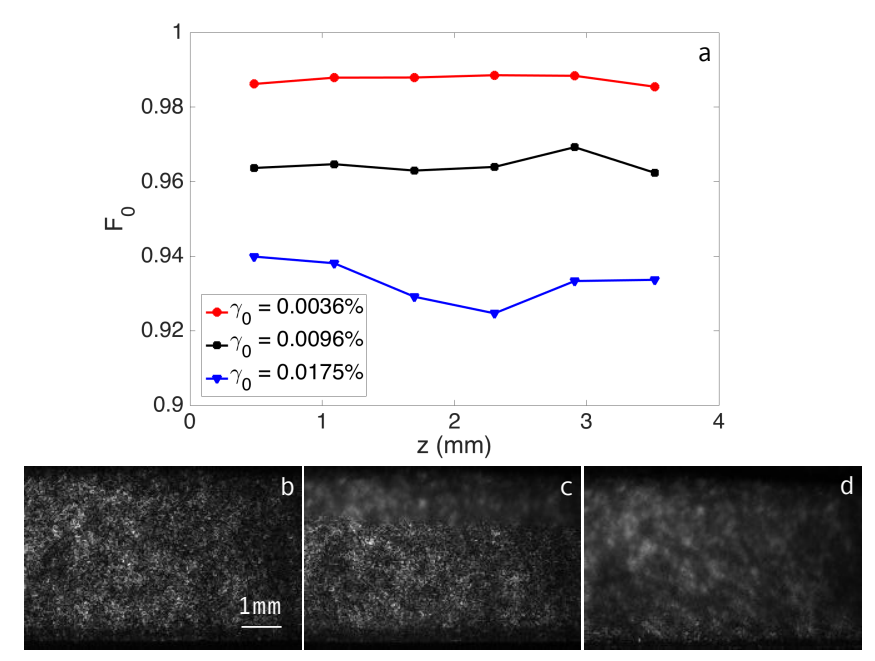

Fig. 6 (a) Optical signal $F_{0}$, as a function of position $z$ in the gap, for various strains in the experiment of Fig. 3. $F_{0}$ denotes the value of $F$ averaged over an oscillation period. (b,c,d) Images of the speckle pattern for applied stresses $\sigma=5 \mathrm{~Pa}(\mathrm{~b})$ and $\sigma=144 \mathrm{~Pa}(\mathrm{c}, \mathrm{d})$. The blurred zone shows where deformation is localized. The shear band is smaller right after the stress has been set to the value $\sigma=144 \mathrm{~Pa}$ (c) than after one minute (d).

\subsubsection{Influence of sample age}

To quantify the aging phenomena, we measured the value of $G^{\prime}$ lin and $\gamma_{\mathrm{cr}}$ for different ages of the same sample. As shown in Fig. 7 we find that the value of $G^{\prime}$ lin only increases of $25 \%$ within one month. Looking at this result and taking into account the value of the standard deviation on $G_{\text {lin }}^{\prime}$ measurement, equal to $15 \%$, we can consider the effect of the aging as non significant. The same conclusion can be drawn from the evolution of $\gamma_{\mathrm{cr}}$.

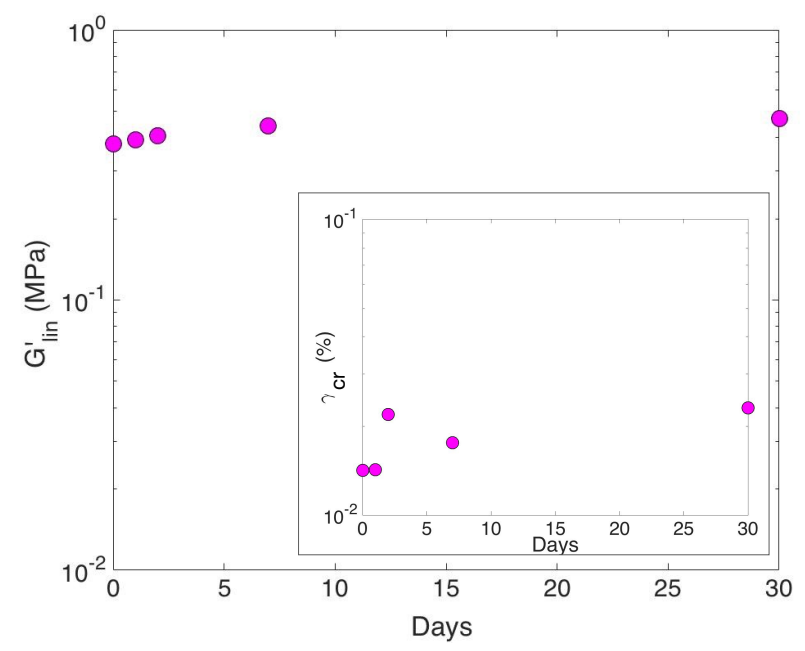

Fig. 7 Aging analysis: linear storage modulus at $20 \%$ volume concentration $\phi$, frequency $1 \mathrm{~Hz}$ and $4 \mathrm{~mm}$ gap. The standard deviation of $G_{\text {lin }}^{\prime}$ is $15 \%$. In the inset is represented the critical strain $\gamma_{\mathrm{cr}}$ in the same conditions with a standard deviation less than $25 \%$. These experiments are performed with $46 \mu \mathrm{m}$-rough sand paper plates.

\subsection{Influence of calcite volume fraction on elasticity and yielding}

Now that we have identified working conditions enabling to avoid the main rheological artifacts, we can focus safely on the influence of the calcite volume fraction $\phi$ on the elastic modulus $G_{l i n}^{\prime}$ and the critical strain for yielding $\gamma_{\mathrm{cr}}$, as shown in Fig. 8 for the frequency $f=1 \mathrm{~Hz}$. In Fig. 8a we see that $G_{\text {lin }}^{\prime}$ constantly rises with $\phi$. In this logarithmic plot, the rise shows a smaller slope at larger concentrations. This change of slope occurs at $\phi \approx 20 \%$. More strikingly, Fig. $8 \mathrm{~b}$ shows that $\gamma_{\mathrm{cr}}$ varies in a non-monotonic way with $\phi$ and exhibits a minimum for $\phi \approx 20 \%$. Finally, the stress at yielding $\tau_{\text {cr }}$ also shows a two-slopes scaling behavior with $\phi$ (Fig. 8c, in logarithmic scales), the change occurring at the same volume fraction.

In the appendix, we also show the data obtained at $f=10 \mathrm{~Hz}$, which exhibit the same behavior.

\section{Discussion}

In the standard fractal elasticity model of colloidal suspensions, proposed initially by Shih et al. ${ }^{6}$, the gel network is viewed as closely packed fractal flocs. A floc is a repetitive highly porous aggregate composed of smaller primary particles ${ }^{36}$. The rigidity and strength of the gel are assumed to be dominated by the weakest element of its network. At low volume fraction, each 

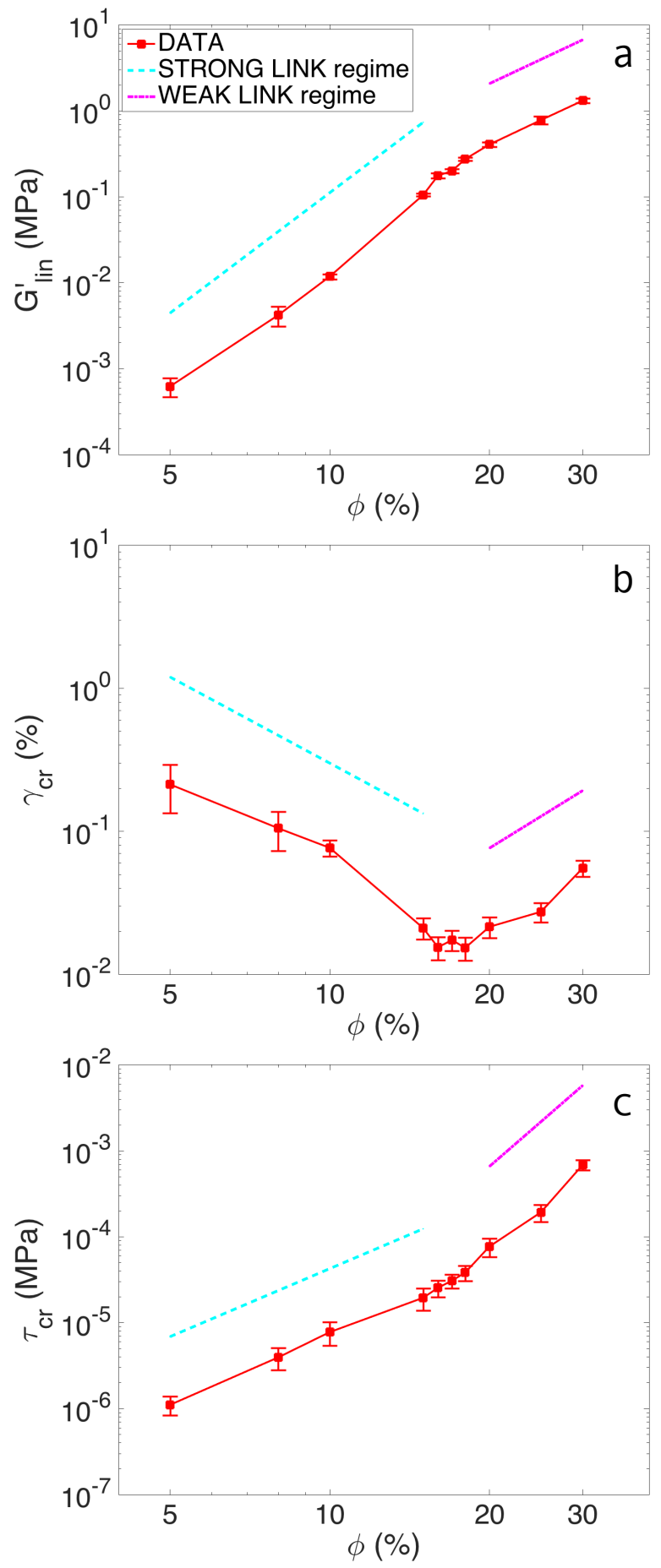

Fig. 8 (a) Linear storage modulus $G_{\text {lin }}^{\prime}$, (b) critical strain $\gamma_{c r}$ and (c) critical stress $\tau_{\mathrm{cr}}$ versus the volume concentration $\phi$ measured at frequency $1 \mathrm{~Hz}$ (red symbols). The blue dashed lines (resp. purple solid lines) show the scaling laws fitted in the strong link (resp. weak link) regime. These experiments are performed with $46 \mu \mathrm{m}$-rough sand paper plates.

floc is highly porous and deformable, and is the weakest element. The inter-floc links are as strong as the flocs, and the regime is called strong-link (SL). Conversely, at higher volume fraction, the flocs are denser and stiffer: the weakest element is therefore the inter-floc link and the regime is called weak-link (WL). Wu and
Morbidelli ${ }^{7}$ re-elaborated this theory introducing an intermediate behavior, called transition regime (TR) to describe continuously the change of mechanical response and scaling laws as a function of the concentration. Several examples of strong-link regime systems are reported: suspensions of alumina ${ }^{6,11}$ or silica $^{10,12}$, nanocomposites made of linear low density polyethylene (LLDPE) and clay ${ }^{9}$ or polyester and exfoliated graphite ${ }^{8}$. Conversely, fewer materials exhibit the weak-link regime like protein solutions ${ }^{17,18}$ or mixture of colloids and polymers ${ }^{19}$. Note that the weak-link scenario is equivalent to double-step yielding, which has been reported for this latter system ${ }^{37,38}$ and attractive microgels ${ }^{39}$ : the first yield point corresponds to the network destruction and formation of individual clusters, and the second yield point to the cluster fragmentation.

Among all the investigated systems, the shift from WL to SL regimes has rarely been observed, and always with changing the external or chemical conditions. One can cite the case of mixture of surfactants and silica colloid, forced to sweep from WL to SL by changing the length of the surfactant hydrophobic chain ${ }^{13}$, magnetorheological fluids, forced by an applied magnetic field ${ }^{14}$, protein gel prepared from eggs white, forced by changing the $\mathrm{pH}^{15}$ and waxy oil system, forced by heating ${ }^{16}$. The model by Shih et al. proposes power law scalings of the elastic modulus $G^{\prime}$, critical strain $\gamma_{\mathrm{cr}}$, and critical stress $\tau_{\mathrm{cr}}$ with $\phi$, as follows:

$$
\begin{aligned}
G^{\prime} & \propto \phi^{A}, \\
\gamma & \propto \phi^{B}, \\
\tau & \propto \phi^{C} .
\end{aligned}
$$

with scaling exponents:

$$
\begin{array}{r}
A=\frac{(d-2)+(2+x)(1-\alpha)}{d-d_{\mathrm{f}}}, \\
B=\frac{1-(2+x)(1-\alpha)}{d-d_{\mathrm{f}}}, \\
C=\frac{d-1}{d-d_{\mathrm{f}}} .
\end{array}
$$

Here $d$ represents the Euclidean dimension (equal to 3 ), $d_{\mathrm{f}}$ the fractal dimension of the flocs and $x$ the fractal dimension of the particle chains (backbone). Note that, from Eq (2), $C$ is equal to $A+B$. The value of $x$ is difficult to estimate and is usually assumed to be in the range of $1-1.3^{40}$. The quantity $\alpha$ is a parameter quantifying the state of the gel, defined by $\mathrm{Wu}$ and Morbidelli ${ }^{7}$, varying from 0 to $1 ; \alpha=0$ corresponds to a SL regime, $\alpha=1$ to a WL regime and intermediate values to TR regimes. If $A$ and $C$ remain positive whatever the value of $\alpha, B$ turns from negative to positive when $\alpha$ changes from 0 to 1 . Thereby, according to this model, the critical strain of a dense colloidal suspension should exhibit a minimum with increasing the concentration.

Quite unexpectedly, despite the wide use of this model, this feature has up to now never been observed experimentally with- 
Table 1 Parameters of the colloidal gel model obtained from the fit of the experimental results for $f=1 \mathrm{~Hz}$ (Fig. $8 \mathrm{a}, \mathrm{b}$ and $\mathrm{c}$ ) with equations 8 and 9 , taking $x=1$.

\begin{tabular}{llllllll}
\hline$\phi$ range & $\alpha$ & A & B & A+B & C & $d_{\mathrm{f}}$ \\
\hline $5 \% \leq \phi \leq 15 \%$ & 0.14 & 4.65 & -2.05 & 2.60 & 2.63 & 2.23 & Regime \\
$15 \% \leq \phi \leq 20 \%$ & 0.69 & 4.50 & 0.19 & 4.69 & 4.64 & 2.57 & strong link \\
$20 \% \leq \phi \leq 30 \%$ & 0.96 & 2.90 & 2.29 & 5.19 & 5.36 & 2.61 \\
\hline
\end{tabular}

Table 2 Parameters of the colloidal gel model obtained from the fit of the experimental results for $f=10 \mathrm{~Hz}$, (Fig. 10b and d) with equations 8 and 9 , taking $x=1$.

\begin{tabular}{lllllll}
\hline$\phi$ range & $\alpha$ & $\mathrm{A}$ & $\mathrm{B}$ & $\mathrm{A}+\mathrm{B}$ & $\mathrm{C}$ & $d_{\mathrm{f}}$ \\
\hline $5 \% \leq \phi \leq 17 \%$ & 0.34 & 4.74 & -1.57 & 3.17 & 3.17 & Regime \\
$17 \% \leq \phi \leq 25 \%$ & 0.67 & 4.68 & -0.05 & 4.63 & 4.77 & 2.37 \\
$25 \% \leq \phi \leq 30 \%$ & 1.06 & 2.30 & 3.39 & 5.69 & 5.59 & strong link \\
\hline
\end{tabular}

out external forcing. Turning to our results, we recognize immediately from Fig. 8 that the mechanical properties of the calcite paste show a power-law behavior changing with concentration exactly as predicted by the colloidal suspension model, with two identified domains separated by a narrow transition zone associated to a minimum of the yield strain observed for $\phi \approx 15-20 \%$. This analysis therefore shows that our system ruptures first inside the flocs at low concentration (SL) and between the flocs at higher concentration (WL).

As a further confirmation of the fact that the calcite paste enters the WL regime, we have performed amplitude sweep experiments up to a $1000 \%$ deformation. We have thereby observed a double-step yielding for $\phi=30 \%$ (see Appendix and Fig. 11). This behavior is characteristic of other attractive weak-link gels ${ }^{37-39}$, where the first yielding, here for $\gamma_{\mathrm{cr}} \sim 0.05 \%$, is a signature of the inter-floc rupture, as predicted by the model, and the second one, here for $\gamma_{0} \sim 5 \%$, corresponds to the fragmentation of the flocs.

The very low value of the first yielding strain $\gamma_{\mathrm{cr}}$ is a further clue of the gel being inside the WL regime. Indeed values of $\gamma_{\mathrm{cr}}$ lower than $0.1 \%$ are characteristic of WL systems, like BSA gels ${ }^{17}$, magnetofluids ${ }^{14}$, or gelled waxy oils ${ }^{16}$, whereas they are unusual in SL sytems.

A second important information can be derived from the evolution of the yield stress with concentration. According to Eq.8c and Eq.9c, the exponent $C$ of the power law evolution of this quantity depends exclusively on $d_{\mathrm{f}}$. Therefore the change of slope shown in Fig.8c should be a consequence of a change of fractal dimension $d_{\mathrm{f}}$ of the gel network with the evolution of the concentration. With this in mind, we have fitted our experimental data of elastic modulus $G_{\text {lin }}^{\prime}$ and yield strain $\gamma_{\mathrm{cr}}$ with Eq (8)a and b, to get exponents $A$ and $B$ in the three domains (SL, TR and WL), choosing $x=1$. We have then solved Eq. (9)a and b, to get $d_{\mathrm{f}}$ and $\alpha$ in each domain. Table 1 shows the values of the exponents of the power laws, of the fractal dimension $d_{\mathrm{f}}$ of the gel, and of the transition parameter $\alpha$ in the 3 concentration domains. Table 2 also shows the results obtained at the frequency $f=10 \mathrm{~Hz}$, which show a very similar behavior. The values of $\alpha$ inform us that, as expected, the low concentration range corresponds to a stronglink regime ( $\alpha \simeq 0$ ), the high concentration range to a weak-link regime ( $\alpha \simeq 1$ ), and the concentrations in-between to a transition regime. The fractal dimension of the gel obtained in the 3 regimes are comparable to values from the literature ${ }^{6,7}$. This dimension is notably larger at higher concentration due to the more crowded structure of the flocs.

The fractal theory proposes a scaling relation between the typical cluster size $D$ and the mean diameter $a$ of the particles which are here assumed to be spherical ${ }^{41}$ :

$$
\phi \sim \phi_{0}(D / a)^{-\left(3-d_{f}\right)}
$$

where $\phi_{0}$ is the maximum packing fraction. With $\phi_{0}=64 \%$ (random close packing) and using the values of $d_{\mathrm{f}}$ from Table 1 , we obtain $D / a=27$ for the extreme value of SL regime and $D / a=7$ for the extreme value of WL. These values give an idea of the structure of the gel network, that we have sketched in Fig. 9.

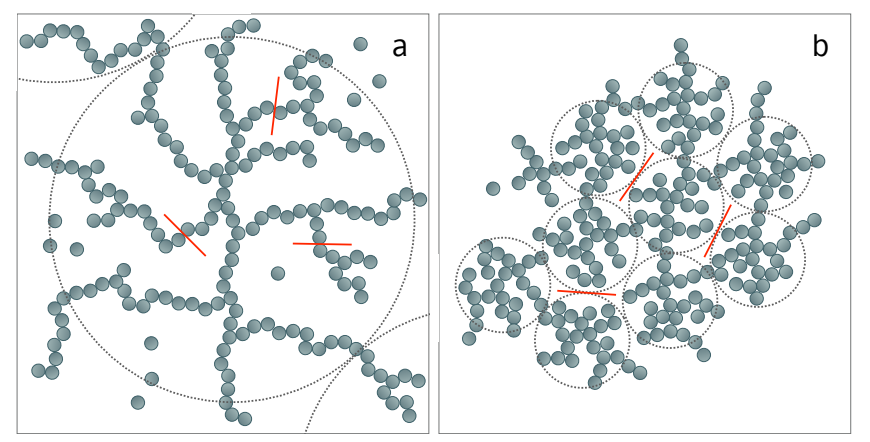

Fig. 9 (a) Strong link structure corresponding to $d_{\mathrm{f}}=2.23$ at low volume fraction, (b) Weak link structure corresponding to $d_{\mathrm{f}}=2.61$ at high volume fraction. Dashed lines represent the flocs at rest and solid lines are the probable rupture points in the two regimes.

The model of Shih et al. ${ }^{6}$ was used numerous times to describe different kinds of colloidal systems. The novelty of our results is to show, for the first time, a complete experimental verification of the model going from the strong link regime to the weaklink regime by increasing the concentration. This verification was carried out with a pure material, varying only the solid particle fraction, without any other external or chemical modification, in contrast with previous works from the literature ${ }^{13-16}$. Moreover the fractal dimension and the size of the flocs extracted thanks to the model in the SL and WL regimes are consistent with what is known from colloidal gels.

The question arises why the calcite paste is the only system 
investigated so far that crosses both regimes. Although it is too early to answer firmly, clues can be found in the literature. In general, large (micron-size) particles are in the SL regime ${ }^{11}$. For small particles $(\sim 10 \mathrm{~nm})$, both regimes are encountered, SL at small concentration $(\phi \leq 10 \%)^{6,12}$ and WL at high concentration $(\phi \geq 15 \%)^{17}$. The calcite particles are small $(\simeq 60 \mathrm{~nm})$, and we find a transition between SL and WL in the range expected from the literature $(\phi \sim 15-20 \%)$. Therefore, it is likely that the calcite paste is the only studied system exhibiting the transition because it is made of particles being both small, and with an attraction strong and short-ranged that enables both to form a gel at low concentration and remain a homogeneous paste at high concentration.

\section{Conclusion}

The mechanical properties of calcite suspensions in water have been investigated through dynamic rheological measurements. The variation of both the elastic modulus in the small deformation linear domain $G_{\text {lin }}^{\prime}$ and the yield strain $\gamma_{\mathrm{cr}}$ (end of the elastic regime) with the solid concentration have been measured. The absence of measurement artifacts such as wall slip effects or heterogeneous deformation fields has been ascertained by optical measurements based on multiple light scattering. The trends of $G_{\text {lin }}^{\prime}$ and $\gamma_{\mathrm{cr}}$ with the solid volume fraction evidence the presence of two different scaling laws: $G_{\text {lin }}^{\prime}$ shows a change in exponent while $\gamma_{\mathrm{cr}}$ goes through a minimum for the same range of solid content.

This feature can be interpreted in the framework of the fractal elasticity model of colloidal suspensions of Shih et al. ${ }^{6,7}$. This model assumes a transition from a regime where elasticity and yielding are dominated by the fractal floc properties at low concentration (strong link regime), to a regime where they are dominated by the links between the flocs (weak link regime), when the flocs become smaller and stiffer as the solid concentration increases. We stress on the fact that this is the first observation of the transition from the strong-link to the weak-link regime in one simple and unperturbed system, predicted by the original model. This interpretation enables to evidence an increase of the fractal dimension of the flocs with increasing solid fraction, pointing to a change of the aggregation with concentration.

This thorough characterization of the elastic and yielding behavior of calcite paste is the first step to understand the particleparticle interactions, and control it through adsorption of organic molecules. This could lead to improve the mechanical properties of building materials such as their ductility. More fundamentally, the flow features (e.g. shear-banding, transient regime or wall slip) rise many open questions which require further studies.

\section{Appendix}

\section{Protocol and frequency dependence}

Fig. 10 presents all the results of the amplitude sweep at different frequencies and experimental conditions. Fig. 10a and c show experiments made at $f=1 \mathrm{~Hz}$ and Fig. $10 \mathrm{~b}$ and $\mathrm{d}$ at $f=10 \mathrm{~Hz}$. As already mentioned, we have verified the gap dependence for the concentration range $\phi=5-30 \%$. For $\phi<10 \%$ only experiments with small gap $(\sim 1 \mathrm{~mm})$ are possible because, for higher gaps, the fluidity of the paste makes it flow outside the gap. In the same figure are also compared the results at $4 \mathrm{~mm}$ gap width and without pre-shear. In most of our experiments, a pre-shear is applied to ensure similar initial conditions and a better reproducibility of the results. However, the few measurements obtained without pre-shear gave very similar results, thus showing a weak effect of the protocol applied to the paste. Concerning the frequency dependence, the results are comparable in term of linear storage modulus $G_{\text {lin }}^{\prime}$, critical strain $\gamma_{\mathrm{cr}}$ and critical stress $\tau_{\mathrm{cr}}$, as a function of the volume concentration $\phi$. For both frequencies, $f=1 \mathrm{~Hz}$ and $f=10 \mathrm{~Hz}$, the transition between strong link to weak ling regime occurs as shown in Fig. 10.

\section{Large amplitude deformation}

To better analyze the yielding and flow mechanism of the calcite paste, we have performed amplitude sweep experiments for deformations up to $1000 \%$ at three different volume concentrations $(10,20$ and 30\%) as shown in Fig. 11. The data obtained at the highest concentration ( $\phi=30 \%$ ) clearly exhibit a two-step yielding, which is consistent with the weak-link scenario for this concentration: the first yield point is associated with the breaking of bonds between clusters, while the second yield point corresponds to the fragmentation of the clusters. However this doubleyielding process has no effect on the study of the onset of plasticity, which is the aim of our study. In the strong-link regime $(\phi=10 \%$ and $20 \%$ ), the cluster destructuration occurs at several length scales so that there is no reason to expect two distinct yield points, consistently with our observations.

\section{Acknowledgements}

This project has received funding from the European Union Horizon 2020 research and innovation program under the Marie Skłodowska-Curie grant agreement No.642976-NanoHeal Project. The results of this paper reflects only the author's view and the Commission is not responsible for any use that may be made of the information it contains. We also thank S. Manneville and B. Saint-Michel for stimulating discussions.

\section{References}

1 A. Putnis, An introduction to mineral sciences, Cambridge University Press, 1992.

2 Mineral and Rocks, ed. J. R. Wilson, Systematic Mineralogy, 2010.

3 P. Coussot, Soft Matter, 2007, 3, 528-540.

4 R. Morini, $P h D$ thesis, Université Nice Sophia Antipolis, 2013.

5 F. Dalas, S. Pourchet, A. Nonat, D. Rinaldi, S. Sabio and M. Mosquet, Cement and Concrete Research, 2015, 71, 115123.

6 W.-H. Shih, W. Y. Shih, S.-I. Kim, J. Liu and I. A. Aksay, Physical Review A, 1990, 42, 4772.

$7 \mathrm{H}$. Wu and M. Morbidelli, Langmuir, 2001, 17, 1030-1036.

8 H. Kim and C. W. Macosko, Macromolecules, 2008, 41, 33173327. 

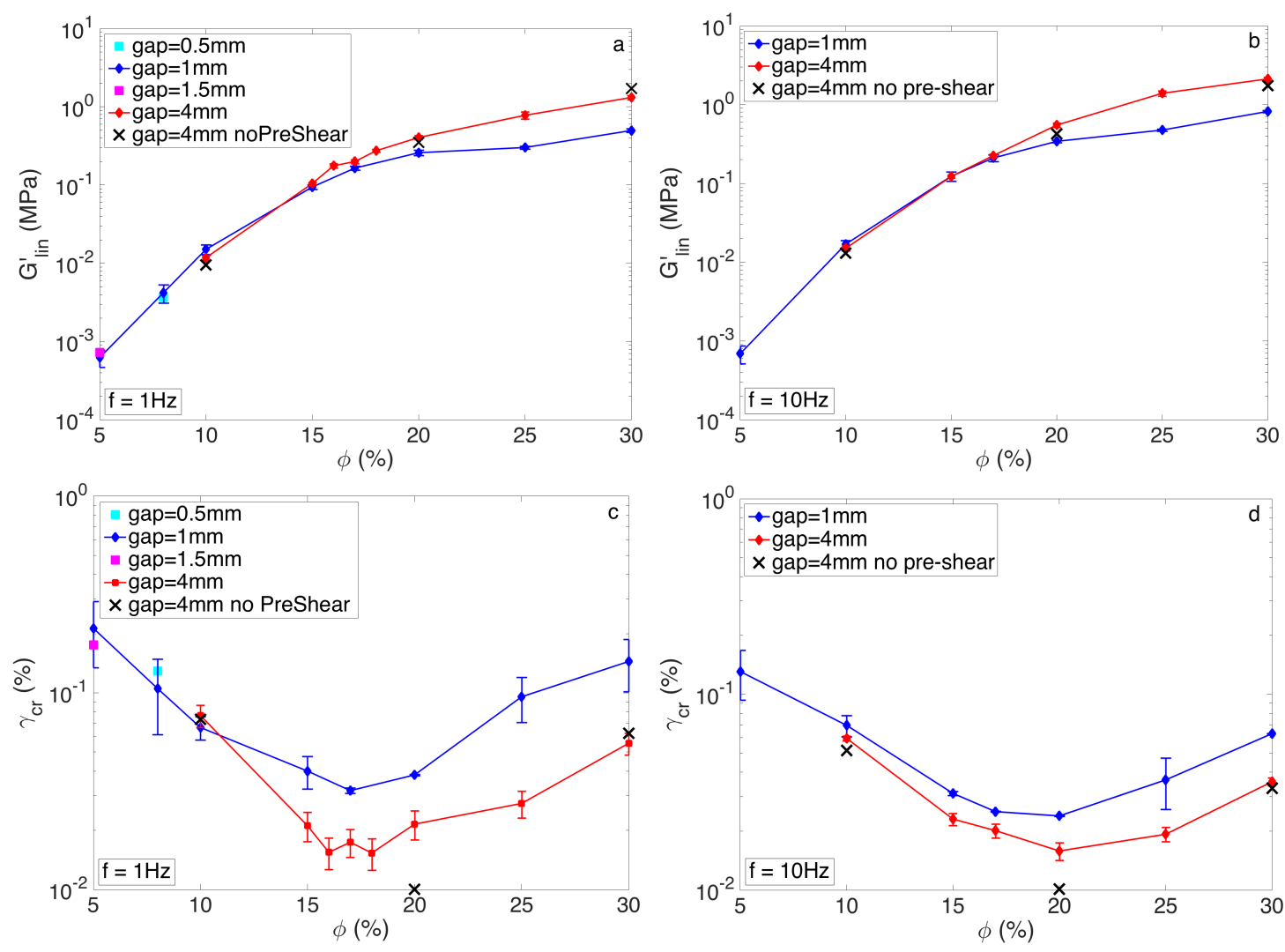

Fig. 10 Influence of gap width and pre-shear: (a) linear storage modulus at frequency $1 \mathrm{~Hz}$, (b) linear storage modulus at frequency $10 \mathrm{~Hz}$, (c) critical strain at $1 \mathrm{~Hz},(\mathrm{~d})$ critical strain at $10 \mathrm{~Hz}$. These experiments are performed with $46 \mu \mathrm{m}$-rough sand paper plates.

9 A. Durmus, A. Kasgoz and C. W. Macosko, Polymer, 2007, 48, 4492-4502.

10 C. Rueb and C. Zukoski, Journal of Rheology, 1997, 41, 197218.

11 G. M. Channell and C. F. Zukoski, AIChE Journal, 1997, 43, 1700-1708.

12 P. Cassagnau, Polymer, 2008, 49, 2183-2196.

$13 \mathrm{H}$. Asai, A. Masuda and M. Kawaguchi, Journal of colloid and interface science, 2008, 328, 180-185.

14 J. Segovia-Gutiérrez, C. Berli and J. De Vicente, Journal of Rheology, 2012, 56, 1429-1448.

15 M. O. Eleya, S. Ko and S. Gunasekaran, Food Hydrocolloids, 2004, 18, 315-323.

16 F. Yang, C. Li, C. Li and D. Wang, Energy \& Fuels, 2013, 27, 3718-3724.

17 T. Hagiwara, H. Kumagai and K. Nakamura, Food Hydrocolloids, 1998, 12, 29-36.

18 S. Ikeda, E. A. Foegeding and T. Hagiwara, Langmuir, 1999, 15, 8584-8589.

19 M. Laurati, G. Petekidis, N. Koumakis, F. Cardinaux, A. B. Schofield, J. M. Brader, M. Fuchs and S. U. Egelhaaf, The Journal of chemical physics, 2009, 130, 134907.

20 A. Kurokawa, V. Vidal, K. Kurita, T. Divoux and S. Manneville, Soft Matter, 2015, 11, 9026-9037.

21 J. Bridge and R. Demicco, Earth surface processes, landforms and sediment deposits, Cambridge University Press, 2008.

22 R. G. Larson, The structure and rheology of complex fluids, Oxford university press New York, 1999, vol. 150.

23 D. A. Weitz and D. J. Pine, in Dynamic Light Scattering, ed. W. Brown, Clarendon, Oxford, 1993, ch. 16, p. 652.

24 L. Cipelletti, H. Bissig, V. Trappe, P. Ballesta and S. Mazoyer, Journal of Physics: Condensed Matter, 2003, 15, S257.

25 A. Duri, D. A. Sessoms, V. Trappe and L. Cipelletti, Physical Review Letters, 2009, 102, 085702.

26 M. Le Merrer, S. Cohen-Addad and R. Höhler, Physical Review Letters, 2012, 108, 188301.

27 X.-L. Wu, D. J. Pine, P. M. Chaikin, J. S. Huang and D. A. Weitz, Journal of the Optical Society of America B, 1990, 7, 15-20.

28 D. Bicout and G. Maret, Physica A, 1994, 210, 87.

29 F. Wintzenrieth, S. Cohen-Addad, M. Le Merrer and R. Höhler, Physical Review E, 2014, 89, 012308.

30 R. H. Ewoldt, M. T. Johnston and L. M. Caretta, Complex Fluids in Biological Systems, Springer, 2015, pp. 207-241.

31 C. Combes, S. Tadier, H. Galliard, S. Girod-Fullana, C. Charvillat, C. Rey, R. Auzély-Velty and N. El Kissi, Acta biomaterialia, 2010, 6, 920-927.

32 J. R. Seth, C. Locatelli-Champagne, F. Monti, R. T. Bonnecaze and M. Cloitre, Soft Matter, 2012, 8, 140-148.

33 T. Gibaud, C. Barentin and S. Manneville, Physical Review Let- 


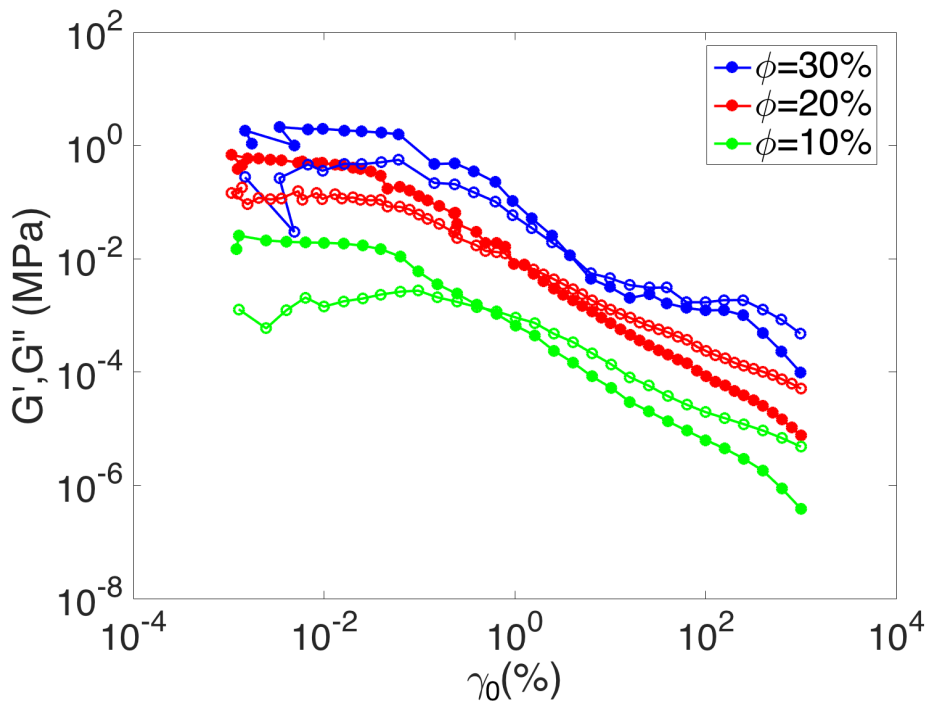

Fig. 11 Amplitude sweep at $30 \%, 20 \%$ and $10 \%$ volume concentration (gap width $4 \mathrm{~mm}, 46 \mu \mathrm{m}$-rough sand paper plates). Full circles represent the $G^{\prime}$ values and the empty circles the $G^{\prime \prime}$ values. For $\phi=30 \%$, in the weak-link regime, a second yield strain can be observed at $\gamma_{0} \simeq 5 \%$.

ters, 2008, 101, 258302.

34 Y. Guo, W. Yu, Y. Xu and C. Zhou, Soft Matter, 2011, 7, 2433.

35 R. Bandyopadhyay, A. S. Gittings, S. S. Suh, P. K. Dixon and D. J. Durian, Review of Scientific Instruments, 2005, 76, 093110.

36 P. Jarvis, B. Jefferson and S. A. Parsons, Reviews in Environmental Science and Bio/Technology, 2005, 4, 1-18.

37 M. Laurati, S. Egelhaaf and G. Petekidis, Journal of Rheology, 2011, 55, 673-706.

38 N. Koumakis and G. Petekidis, Soft Matter, 2011, 7, 24562470.

39 Z. Shao, A. S. Negi and C. O. Osuji, Soft Matter, 2013, 9, 5492.

40 A. G. Marangoni et al., Fat crystal networks, 2005, 413-440.

41 R. Buscall, P. D. Mills, J. W. Goodwin and D. Lawson, Journal of the Chemical Society, Faraday Transactions 1: Physical Chemistry in Condensed Phases, 1988, 84, 4249-4260. 